Weiterentwicklung) als Studienfach an niederländischen und belgischen Universitäten.

Willem F. Daems

R. Hooykaas, G. J.Rheticus' Treatise on Holy Scripture and the Motion of the Earth with translation, annotations, commentary and additional chapters of Ramus-Rheticus and the development of the problems before 1650 ( = Verhandelingen der Koninklijke Nederlandse Akademie van Wetenschappen, Afd. Letterkunde, Nieuwe Reeks, 124). 190 S. North-Holland Publishing Company, Amsterdam, Oxford, New York 1984.

Der niederländische Wissenschaftshistoriker Hooykaas identifizierte eine bislang unbekannt gebliebene Abhandlung über die Bewegung der Erde als eine Schrift des George Joachim Rheticus (nach 1532 geschrieben). Dieser ein direkter Schüler des Kopernikus - verteidigt darin seinen Meister gegen die Anschuldigung, seine Theorie über die Erdbewegung sei im Konflikt mit der Autorität der Bibel. Hooykaas beschreibt zunächst, wer Rheticus war, wie er ihn als Verfasser der Arbeit identifizieren konnte und wann die Schrift abgefaßt wurde. Der Hauptteil bringt eine Übersetzung in englischer Sprache. Dann folgt ein ausführlicher Apparat mit Anmerkungen, Referenzen, Kommentaren, Belegstellen aus der Bibel und aus Werk von Augustinus. Spezielle Probleme - u. a. aus der Beziehung von Rheticus zu Petrus Ramus und das des Druckes der anonymen Abhandlung - werden in den letzten zwei Kapiteln abgehandelt.

Willem F. Daems

\title{
Buchanzeigen / Book Notices
}

ZMA $=$ Zürcher medizingeschichtliche Abhandlungen, alle aus dem Juris-Verlag, Zürich

Peter Zupan, der Physiologe Carl Ludwig in Zürich 1849-1855 (ZMA Nr. 188). Außer einem biographischen Überblick werden vor allem die Jahre 1849-1855 dargestellt, welche Ludwig als Professor der Anatomie und Physiologie an der Universität Zürich zubrachte.

Daniela à Wengen-Dörig, Die Geschichte der manuellen und instrumentellen Beckenmessung 1650-1886 (ZMA Nr. 189). Die Pelvimetrie entstand im Laufe des 18. Jahrhunderts und verlor wieder an Bedeutung durch die Einführung der Röntgendiagnostik. Die Abhandlung wird ergänzt durch zahlreiche Abbildungen von ehemals verwendeten Instrumenten.

Rainer Otto Hardegger, Die Helvetische Gesellschaft correspondierender Ärzte und Wundärzte 1788/91-1807 (ZMA Nr. 191). Darstellung der Entstehung der ersten schweizerischen Ärztevereinigung. 
Albert Steiner, Ludwig Choulant und seine "Anleitung zu dem Studium der Medicin» (1829) (ZMA Nr.192). Die Abhandlung resümiert das damals viel gelesene Büchlein des bekannten Klinikers Choulant. Sie vergleicht das Werk mit der «Einleitung in das Studium der Medicin», von Johann W. H.Conradi, die ein Jahr zuvor erschienen war.

Roland Kunz, Der Kinderarzt Emil Feer (1864-1955) (ZMA Nr. 193). Feer begründete das Spezialfach der Pädiatrie in Zürich und schuf das erste Kinderspital dieser Stadt. In kurzen Biographien werden auch seine wichtigsten Schüler gewürdigt.

Barbara Ruth Wendel-Widmer, Die Wunderheilungen am Grabe der Heiligen Elisabeth von Thüringen (ZMA Nr.194). Darstellung des breiten Spektrums von Erkrankungen, die Anlaß gaben zu einer Pilgerfahrt an das Grab der hl. Elisabeth, sowie eine Aufschlüsselung der Pilgerschaft nach ihrer sozialen Herkunft.

Shin'ichirô Tomonaga, Welt im Spiegel. Franz Steiner Verlag Wiesbaden GmbH, Stuttgart 1986. Der japanische Theoretische Physiker, der 1965 den Nobelpreis erhielt, schreibt hier Erinnerungen an seine Studienzeit, wovon 1938/39 bei Heisenberg in Leipzig sowie Betrachtungen über seine Erfahrungen als Universitätsdozent und -rektor.

Rudi von Poldenko, Sektionsprotokolle. Glossen und Satiren. Deutscher Ärzte Verlag, Köln 1987. Texte, die fast alle im Deutschen Ärzteblatt erschienen und hier in einem Büchlein gesammelt herausgegeben wurden. Der Autor karikiert vor allem den Wissenschaftsbetrieb, die Publikationsflut und den Kongreßtourismus.

\section{Adressen der Rezensenten}

Dr. phil. Willem F. Daems, Weleda, CH-4144 Arlesheim

Dr. J. Druey, Schnitterweg 52, CH-4125 Riehen

Prof. Dr. med.C. Haffter, Petersgasse 50, CH-4051 Basel

Prof. Dr. H. Haffter, Sträulistraße 4, CH-8400 Winterthur

Dr. F. Ledermann, Könizstraße 3, CH-3008 Bern

Dr. E. Neuenschwander, Meientalstraße 71, CH-8048 Zürich

PD Dr.phil. O. Rieppel, Paläontologisches Institut, Künstlergasse 16, CH-8006 Zürich

PD Dr. sc.nat. Dr. phil.G. Schramm, Waidspital, Tièchestraße 99, CH-8037 Zürich

Dr. med. A. Stettler, Kirchenfeldstraße 45, CH-3006 Bern

Redaktionsschluß für die nächsten Hefte des Gesnerus:

- 27. Februar 1989 für Heft 1/2 1989 (erscheint am 31. Mai 1989)

- 29. August 1989 für Heft 3/4 1989 (erscheint am 30. November 1989) 